\title{
Hemodiálise: análise da qualidade de vida de indivíduos renais crônicos
}

\author{
Hemodialysis: quality of life analysis of chronic kidney individuals
}

Pollyanna Silva ${ }^{1}$, Jacqueline Andréia Bernardes Leão-Cordeiro²; Rogério José de Almeira $^{3}$; Antonio Márcio Teodoro Cordeiro Silva ${ }^{4}$

\section{RESUMO}

A doença renal crônica tem alta morbimortalidade e gera grande impacto no cotidiano e na qualidade de vida dos pacientes. $O$ artigo objetivou avaliar a qualidade de vida de portadores de doença renal crônica, em hemodiálise. Tratou-se de um estudo transversal analítico com abordagem quantitativa, realizado em clínica de hemodiálise, em Porangatu/GO, com 102 pacientes, em tratamento hemodialítico, utilizando o instrumento KDQOL-SF e questionário socioeconômico-demográfico. Como resultados, verificou-se que, nas dimensões genéricas, o maior escore foi o bem-estar emocional, com média de 68,51 (72,12 para homens e 61,6 para mulheres). Outros escores significativos foram: função social $(63,73)$ e energia e fadiga $(61,18)$. Os menores escores foram: função física $(12,99)$ e função emocional $(20,59)$. Nas dimensões específicas do KDQOL-SF, os maiores escores foram: estímulo por parte da equipe de diálise $(95,96)$ e satisfação do paciente $(90,36)$. Os menores escores foram: papel profissional $(7,35)$ e sobrecarga da doença renal $(37,56)$. Concluiu-se que a maioria dos pacientes era constituída de homens, com renda e escolaridade baixas, hipertensos e diabéticos, em uso de medicação; e a qualidade de vida se mostrou mais afetada nas dimensões: "papel profissional" e "função física". Identificar fatores determinantes para a melhora do quadro clínico, possibilita subsídios na implementação de novas estratégias eficientes no ensino, na prática e em estudos relacionados à pessoa com doença renal crônica, em hemodiálise, visando melhoria das suas condições de vida.

Palavras-chave: Doença renal crônica. Qualidade de vida. Hemodiálise. KDQOL-SF.

\section{ABSTRACT}

Chronic kidney disease has a high morbidity and mortality and has a great impact on patients' daily lives and quality of life. The article aimed to evaluate the quality of life of patients with chronic kidney disease undergoing hemodialysis. This was an analytical cross-sectional study with a quantitative approach, carried out in a hemodialysis clinic in Porangatu/GO, with 102 patients undergoing hemodialysis, using the KDQOL-SF instrument and a socioeconomic-demographic questionnaire. As a result, it was found that, in the generic dimensions, the highest score was emotional well-being, with an average of 68.51 (72.12 for men and 61.6 for women). Other significant scores were social function (63.73) and energy and fatigue (61.18). The lowest scores were physical function (12.99) and emotional function (20.59). In the specific dimensions of the KDQOL-SF, the highest scores were encouragement by the dialysis team (95.96) and patient satisfaction (90.36). The lowest scores were professional role (7.35) and kidney disease burden (37.56). It was concluded that most patients were men, with low income and education, hypertensive, and diabetic patients, using medication; and the quality of life was more affected in the dimensions: "professional role" and "physical function". Identifying determinant factors for the improvement of the clinical condition, allows subsidies in the implementation of new efficient strategies in teaching, in practice and in studies related to people with chronic kidney disease, on hemodialysis, aiming at improving their living conditions.
'Mestre, Programa de PósGraduação em Ciências Ambientais e Saúde (MCAS), Pontifícia Universidade Católica de Goiás (PUC Goiás), Goiânia, Goiás, Brasil.

E-mail:

pollyanna_silva@hotmail.com ORCID: $\quad$ https://orcid.org/00000001-5079-3983

${ }^{2}$ Doutora. Faculdade Enfermagem (FEN), Universidade Federal de Goiás (UFG), Goiânia, Goiás, Brasil.

ORCID: https://orcid.org/00000003-0703-3609

${ }^{3}$ Doutor. Curso de Medicina (MED), Escola de Ciências Médicas, Farmacêuticas

Biomédicas (EMFB), Pontifícia Universidade Católica de Goiás (PUC Goiás), Goiânia, Goiás, Brasil.

ORCID: https://orcid.org/00000002-2150-6057

${ }^{4}$ Doutor. Programa de PósGraduação em Ciências Ambientais e Saúde (MCAS), Curso de Medicina (MED), Escola de Ciências Médicas, Farmacêuticas e Biomédicas (EMFB), Pontifícia Universidade Católica de Goiás (PUC Goiás), Goiânia, Goiás, Brasil.

ORCID: https://orcid.org/00000003-0645-3599

Keywords: Chronic kidney disease. Quality of life. Hemodialysis. KDQOL-SF. 


\section{INTRODUÇAOO}

A doença renal crônica (DRC) consiste em lesão renal e perda progressiva e irreversível da função dos rins (glomerular, tubular e endócrina). Na fase mais avançada, chamada de fase terminal da insuficiência renal crônica (IRC), os rins são incapazes de manter a normalidade das condições internas dos indivíduos. A hemodiálise é o tratamento de primeira escolha. Por outro lado, no transcorrer dos anos, essa terapia afeta outros fatores fisiológicos, que pode desencadear danos: cardíacos, pulmonares, vasculares, como congestão pulmonar, sendo a mais frequente, relacionados ao distúrbio restritivo, a redução de fluxo de ar para o teste de função pulmonar (MAFRA et al., 2019).

De acordo com o último censo da Sociedade Brasileira de Nefrologia (SBN), do ano de 2016, o número total estimado de pacientes em tratamento hemodialítico, no país, era de 122.825. Este número representa um aumento de 31,5 mil pacientes nos últimos 5 anos (91.314, em 2011) (SESSO et al., 2017). No Brasil, mais de 100.000 pessoas são dependentes de tratamento hemodialítico e 30\% têm mais de 65 anos. Em torno de 90\% estão em hemodiálise, sendo que $85 \%$ desse tratamento financiado pelo Sistema Único de Saúde (SUS), com um gasto anual estimado em $R$ \$ 2,2 bilhões (GOUVEIA et. al., 2017).

O procedimento hemodialítico pode causar limitações e prejuízos nos estados de saúde mental, física, funcional, de bem-estar e social. Na grande maioria, essas limitações e frustações acontecem pelo fato de serem acompanhadas de diversas restrições, como manutenção de uma dieta específica e, principalmente, modificação na aparência corporal em detrimento da presença do cateter para acesso vascular ou da fístula arteriovenosa. Portanto, vários aspectos inerentes ao tratamento afetam a qualidade de vida dos portadores de doença renal crônica (BETTONI; OTTAVIANI; ORLANDI, 2017; DIPP et al., 2019).

Portanto, o objetivo deste estudo foi avaliar a qualidade de vida de indivíduos renais crônicos em tratamento de hemodiálise.

\section{MATERIAIS E METODOS}

Estudo transversal analítico com abordagem quantitativa. Participaram 102 pacientes portadores de DRC em uma clínica de hemodiálise localizada na cidade de Porangatu/GO.

Foram considerados critérios de inclusão: ter idade igual ou maior que 18 anos; ter o diagnóstico de doença renal crônica; ser capaz de compreender os objetivos do estudo e de responder verbalmente às perguntas; estar em hemodiálise há, pelo menos, 90 dias. 
Como critérios de exclusão, foram considerados: pacientes em trânsito na clínica, que não estavam cadastrados; ter algum comprometimento neurológico que pudesse afetar a coleta; condição de instabilidade hemodinâmica no período da coleta.

Para o desenvolvimento da pesquisa foi utilizado instrumentos: questionário socioeconômico, demográfico e de saúde. Este instrumento de pesquisa abordou as seguintes variáveis: sexo, idade, renda pessoal, escolaridade, comorbidades, uso de medicamentos regulares, antecedentes familiares, hábitos de vida, atividade física, deslocamento para clínica de hemodiálise, plano de hemodiálise e alimentação com legumes, verduras e frutas, mudanças no estilo de vida devido à hemodiálise.

Foi utilizado também o instrumento Kidney Disease and Quality of Live Short Form (KDQOL-SF). Este instrumento foi desenvolvido por Working Group, em 1997 (versão 1.3), e validado no Brasil, em 2003 (DUARTE; CICONELLI; SESSO, 2005). O KDQOL-SF é aplicável a pacientes em hemodiálise e tem como objetivo mensurar a qualidade de vida em pacientes renais crônicos em suas especificidades.

Os dados obtidos foram compilados em planilhas eletrônicas e analisados estatisticamente, com auxílio do software BioEstat 5.3. Foi realizada estatística descritiva com o cálculo das medidas de tendência central para as variáveis contínuas, como média e mediana, e de dispersão, como desvio padrão, e cálculo das frequências absoluta e relativa percentual para as variáveis categóricas. As respostas do KDQOL-SF foram convertidas em escores e recodificadas, seguindo as recomendações do KDQOL Working Group (HAYS et al., 1997). Na sequência, foi aplicado teste de normalidade (KolmogorovSmirnov). Foram utilizados, para as distribuições paramétricas, os testes $t$ de Student e ANOVA e para as distribuições não-paramétricas os testes Mann-Whitney e Kruskal-Wallis. Foi adotado o nível de significância de 5\%.

A presente pesquisa foi encaminhada ao Comitê de Ética em Pesquisa da Pontifícia Universidade Católica de Goiás (PUC Goiás), recebendo aprovação (CAAE: 02670318.7.0000.0037).

\section{RESULTADOS}

Participaram 102 indivíduos, com DRC, em tratamento hemodialítico, com média de idade de $58,1( \pm 15,3)$ anos (19 a 91 anos). Destes, $65,7 \%$ eram indivíduos do sexo masculino. Em relação à renda pessoal, a maioria $(76,5 \%)$ contava com até um salário mínimo e 4,9\% declararam não possuir nenhuma renda. Sobre a escolaridade, 19,6\% 
admitiram nunca ter estudado e a maioria dos participantes $(56,8 \%)$ cursou o ensino fundamental. Quando indagados sobre a moradia, 80,4\% declararam possui casa própria (Tabela 1). Sobre as comorbidades, evidenciadas na pesquisa, a hipertensão arterial foi a mais prevalente $(68,6 \%)$, seguida do diabetes mellitus $(37,3 \%)$ (Tabela 2$)$.

Em relação aos medicamentos de uso regular, 95,1\% afirmaram utilizar algum medicamento. O medicamento mais utilizado pelos pacientes foi a Losartana $(23,5 \%)$ (Tabela 3). Sobre os antecedentes familiares, os principais foram: a hipertensão arterial sistêmica (67,6\%) e o diabetes mellitus. A doença renal acometeu 20,6\% dos familiares dos participantes. Em relação aos hábitos do tabagismo e do etilismo, a maioria não fumava $(95,1 \%)$ nem ingeria bebida alcoólica $(94,1 \%)$, porém $76,5 \%$ admitiram não praticar atividade física regularmente (Tabela 4).

Tabela 1. Dados socioeconômico-demográficos dos 102 pacientes portadores de DRC na Clínica de Hemodiálise, Porangatu, Goiás, Brasil, 2019.

\begin{tabular}{lcc}
\hline Variáveis (n=102) & $\boldsymbol{n}$ & $\boldsymbol{f}(\%)$ \\
\hline Sexo & 67 & 65,7 \\
$\quad$ Masculino & 35 & 34,3 \\
Feminino & & \\
Idade (anos) & 52 & 51,0 \\
$\quad$ Até 59 anos & 50 & 49,0 \\
$\geq 60$ anos & 58,1 & 15,3 \\
Média (DP) & 19 & 91 \\
Mín - Máx & & \\
Renda Pessoal & 5 & 4,9 \\
Sem Renda & 78 & 76,5 \\
Até 1 SM & 16 & 15,7 \\
2 SM & 1 & 1,0 \\
3 SM & 2 & 2,0 \\
Acima de 3 SM & & \\
Escolaridade & 20 & 19,6 \\
Nunca Estudei & 34 & 33,3 \\
Fundamental Incompleto & 24 & 23,5 \\
Fundamental Completo & 9 & 8,8 \\
Médio Incompleto & 12 & 11,8 \\
Médio Completo & 1 & 1,0 \\
Superior Incompleto & 2 & 2,0 \\
Superior Completo & & 80,4 \\
Casa Própria & 82 & 19,6 \\
Sim & 20 &
\end{tabular}


Tabela 2. Comorbidades dos 102 pacientes portadores de DRC na Clínica de Hemodiálise, Porangatu, Goiás, Brasil, 2019.

\begin{tabular}{lcc}
\hline Variáveis $(\boldsymbol{n = 1 0 2})$ & $\boldsymbol{n}$ & $\mathbf{f ( \% )}$ \\
\hline Hipertensão Arterial & 70 & 68,6 \\
Sim & 32 & 31,4 \\
Não & & \\
Diabetes Mellitus & 38 & 37,3 \\
Sim & 64 & 62,7 \\
Não & & \\
Hipercolesterolemia & 9 & 8,8 \\
Sim & 93 & 91,2 \\
Não & & \\
Obesidade & 7 & 6,9 \\
Sim & 95 & 93,1 \\
Não & & 18,6 \\
Outras & 19 & 81,4 \\
Sim & 83 & \\
Não & & \\
\hline
\end{tabular}

Tabela 3. Caracterização medicamentosa dos 102 pacientes portadores de DRC na Clínica de Hemodiálise, Porangatu, Goiás, Brasil, 2019.

\begin{tabular}{|c|c|c|}
\hline Variáveis $(n=102)$ & $n$ & $f(\%)$ \\
\hline \multicolumn{3}{|c|}{ Medicamento Regular } \\
\hline $\operatorname{Sim}$ & 97 & 95,1 \\
\hline Não & 5 & 4,9 \\
\hline \multicolumn{3}{|l|}{ Losartana } \\
\hline Sim & 24 & 23,5 \\
\hline Não & 78 & 76,5 \\
\hline \multicolumn{3}{|l|}{ Nifedipina } \\
\hline Sim & 14 & 13,7 \\
\hline Não & 88 & 86,3 \\
\hline \multicolumn{3}{|l|}{ Atenolol } \\
\hline Sim & 12 & 11,8 \\
\hline Não & 90 & 88,2 \\
\hline \multicolumn{3}{|l|}{ Insulina NPH } \\
\hline Sim & 10 & 9,8 \\
\hline Não & 92 & 90,2 \\
\hline \multicolumn{3}{|l|}{ Atensina } \\
\hline Sim & 6 & 5,9 \\
\hline Não & 96 & 94,1 \\
\hline \multicolumn{3}{|l|}{ Insulina regular } \\
\hline Sim & 5 & 4,9 \\
\hline Não & 97 & 95,1 \\
\hline \multicolumn{3}{|l|}{ Captopril } \\
\hline Sim & 2 & 2,0 \\
\hline Não & 100 & 98,0 \\
\hline
\end{tabular}




\section{Glibencamida}

$\begin{array}{lcc}\text { Sim } & 2 & 2,0 \\ \text { Não } & 99 & 97,1\end{array}$

\section{Metformina}

Sim

Não

100

98,0

Propranolol

Sim

$$
2
$$

Não

\section{Enalapril}

Sim

Tabela 4. Antecedente familiares e hábitos dos 102 pacientes portadores de DRC na Clínica de Hemodiálise, Porangatu, Goiás, Brasil, 2019.

Variáveis $(n=102)$ n

\section{Antecedentes familiares}

HAS

Sim

Não

DM

Não

\section{Obesidade}

Sim

25,5

Não

Hipercolesterolemia

Sim

Não

\section{Doença Renal}

Sim

Não

\section{Outros}

Sim

Não

Tabagismo

Sim

Não

\section{Etilismo}

Sim

Não

\section{Atividade Física}

Sim

Não 
Sobre ao deslocamento para a unidade de hemodiálise, a maior parte (54,9\%) contava com a Van para chegar até o local. Em relação ao plano de saúde utilizado, verificou-se que $98,0 \%$ faziam hemodiálise pelo SUS. Sobre hábitos alimentares dos pacientes, evidenciou-se que o consumo de legumes e verduras $(98,0 \%)$ e frutas $(96,1 \%)$ ocorre, geralmente, com a frequência superior a três vezes por semana (Tabela 5). E em relação ao conhecimento acerca da sua própria saúde, 90,2\% dos entrevistados afirmaram conhecer. Sobre o entendimento da função da hemodiálise, 95,1\% concordaram saber. Um total de $96,1 \%$ percebeu mudanças no estilo de vida com a hemodiálise, especialmente no trabalho $(96,1 \%)$ (Tabela 6).

Tabela 5. Características da hemodiálise dos 102 pacientes portadores de DRC na Clínica de Hemodiálise, Porangatu, Goiás, Brasil, 2019.

\begin{tabular}{|c|c|c|}
\hline Variáveis $(n=102)$ & $n$ & $f(\%)$ \\
\hline \multicolumn{3}{|l|}{ Vai para e Hemodiálise } \\
\hline Van & 56 & 54,9 \\
\hline Ambulância & 10 & 9,8 \\
\hline Carro Próprio & 7 & 6,9 \\
\hline Moto & 7 & 6,9 \\
\hline Outros & 12 & 11,8 \\
\hline Não Respondeu & 10 & 9,8 \\
\hline \multicolumn{3}{|l|}{ Faz Hemodiálise pelo } \\
\hline SUS & 100 & 98,0 \\
\hline Convênios & 2 & 2,0 \\
\hline Particular & 0 & 0,0 \\
\hline \multicolumn{3}{|l|}{ Legumes e Verduras } \\
\hline Sim & 100 & 98,0 \\
\hline Não & 2 & 2,0 \\
\hline \multicolumn{3}{|l|}{ Com que Frequência? } \\
\hline Menos de 2x/Semana & 13 & 12,7 \\
\hline $2 x /$ Semana & 17 & 16,7 \\
\hline 3X/Semana & 32 & 31,4 \\
\hline Mais de $3 x /$ Semana & 38 & 37,3 \\
\hline Não Respondeu & 2 & 2,0 \\
\hline \multicolumn{3}{|l|}{ Frutas } \\
\hline $\operatorname{Sim}$ & 98 & 96,1 \\
\hline Não & 4 & 3,9 \\
\hline \multicolumn{3}{|l|}{ Com que Frequência? } \\
\hline Menos de 2x/Semana & 20 & 19,6 \\
\hline $2 x /$ Semana & 21 & 20,6 \\
\hline 3X/Semana & 30 & 29,4 \\
\hline Mais de $3 x /$ Semana & 27 & 26,5 \\
\hline Não Respondeu & 4 & 3,9 \\
\hline
\end{tabular}


Tabela 6. Saúde, hemodiálise e atividades cotidianas dos 102 pacientes portadores de DRC na Clínica de Hemodiálise, Porangatu, Goiás, Brasil, 2019.

\begin{tabular}{|c|c|c|}
\hline Variáveis $(n=102)$ & $n$ & $f(\%)$ \\
\hline \multicolumn{3}{|c|}{ Sabe sobre sua Saúde } \\
\hline Sim & 92 & 90,2 \\
\hline Não & 10 & 9,8 \\
\hline \multicolumn{3}{|c|}{ Função da Hemodiálise } \\
\hline Sim & 97 & 95,1 \\
\hline Não & 5 & 4,9 \\
\hline \multicolumn{3}{|c|}{ Mudanças com a Hemodiálise } \\
\hline Sim & 98 & 96,1 \\
\hline Não & 4 & 3,9 \\
\hline \multicolumn{3}{|l|}{ No trabalho } \\
\hline $\operatorname{Sim}$ & 98 & 96,1 \\
\hline Não & 4 & 3,9 \\
\hline \multicolumn{3}{|c|}{ Atividades do Dia-a-Dia } \\
\hline Sim & 97 & 95,1 \\
\hline Não & 5 & 4,9 \\
\hline \multicolumn{3}{|l|}{ Viagens } \\
\hline Sim & 96 & 94,1 \\
\hline Não & 6 & 5,9 \\
\hline \multicolumn{3}{|l|}{ Lazer } \\
\hline $\operatorname{Sim}$ & 92 & 90,2 \\
\hline Não & 10 & 9,8 \\
\hline \multicolumn{3}{|l|}{ Passeios } \\
\hline Sim & 91 & 89,2 \\
\hline Não & 11 & 10,8 \\
\hline \multicolumn{3}{|c|}{ Na relação com meu parceiro } \\
\hline Sim & 63 & 61,8 \\
\hline Não & 39 & 38,2 \\
\hline \multicolumn{3}{|c|}{ Relação com os familiares } \\
\hline Sim & 53 & 52,0 \\
\hline Não & 49 & 48,0 \\
\hline \multicolumn{3}{|l|}{ Na escola } \\
\hline Sim & 12 & 11,8 \\
\hline Não & 90 & 88,2 \\
\hline
\end{tabular}

Nas dimensões genéricas do KDQOL-SF, o escore com melhor desempenho foi o bem-estar emocional $(68,51)$. Outros escores, com bom desempenho, foram: função social $(63,73)$, energia e fadiga $(61,18)$ e dor $(55,59)$. Os menores escores médios obtidos foram: funcionamento físico $(40,05)$, função emocional $(20,59)$ e função física $(12,99)$. Os escores gerais, os de pior desempenho foram: função física $(12,99)$ e função emocional $(20,59)$.

Nas dimensões específicas do KDQOL-SF, os maiores escores médios foram: estímulo por parte da equipe de diálise $(95,96)$, satisfação do paciente $(90,36)$, função 
cognitiva $(78,56)$ e qualidade da interação social $(75,69)$. Os menores escores médios obtidos foram no papel profissional $(07,35)$, na sobrecarga da doença renal $(37,56)$ e efeitos da doença renal $(48,74)$.

A comparação entre as médias dos escores para homens e mulheres apresentou três escores estatisticamente distintos, com os homens com escores maiores que as mulheres. As dimensões foram: bem-estar emocional $(72,12 \times 61,60 ; p=0,0197)$; lista de sintomas e problemas $(72,12 \times 69,41 ; p=0,0435)$; e, finalmente, função cognitiva $(82,79 \times$ 70,48; $p=0,0151$ ) (Tabela 7).

A Tabela 8 mostra os escores das 19 dimensões do KDQOL-SF estratificadas pelas variáveis sociodemográficas e clínicas. As variáveis com diferenças estatísticas significantes em maior número de dimensões foram: diabetes mellitus, com 13 dimensões diferentes e hipertensão arterial sistêmica, com 11 dimensões distintas.

No caso do diabetes mellitus, as dimensões afetadas foram: funcionamento físico ( $p=0,0016)$; função física ( $p=0,0349)$; saúde geral $(p=0,0091)$; bem-estar emocional ( $p=0,0308)$; função emocional $(p=0,0303)$; função social $(p=0,0039)$; energia e fadiga ( $p=0,0027)$; lista de sintomas e problemas $(p=0,0024)$; efeitos da doença renal $(p=0,0012)$; função cognitiva ( $p=0,0052)$; qualidade da interação social ( $p=0,0351)$; função sexual $(p=0,0068)$; e sono $(p=0,0392)$.

Pacientes com hipertensão arterial sistêmica apresentam as seguintes dimensões afetadas: saúde geral ( $p=0,0005)$; bem-estar emocional $(p=0,0055)$; função emocional ( $p=0,0482)$; função social ( $p=0,0142)$; energia e fadiga $(p=0,0038)$; lista de sintomas e problemas $(p=0,0264)$; efeitos da doença renal $(p=0,0009)$; função cognitiva $(p=0,0083)$; qualidade da interação social $(p=0,0213)$; função sexual $(p=0,0453)$; e satisfação do paciente $(p=0,0201)$ (Tabela 8$)$. 
Tabela 7. Dimensões do KDQOL-SF (Kidney Disease Quality of Life Short Form) dos 102 pacientes portadores de DRC na Clínica de Hemodiálise, Porangatu, Goiás, Brasil, 2019.

\begin{tabular}{|c|c|c|c|c|c|c|c|c|c|c|c|c|}
\hline \multirow{3}{*}{\multicolumn{2}{|c|}{ Escala ou Dimensão }} & \multicolumn{3}{|c|}{ Geral $(n=102)$} & \multicolumn{3}{|c|}{ Masculino $(n=67)$} & \multicolumn{3}{|c|}{ Feminino $(n=35)$} & \multirow{3}{*}{$p$-valor } & \multirow{3}{*}{$\begin{array}{c}\text { Alfa de } \\
\text { Cronbach }\end{array}$} \\
\hline & & \multirow{3}{*}{$\begin{array}{l}\text { Média } \\
40,05\end{array}$} & \multicolumn{2}{|c|}{ IC95\% } & \multirow{3}{*}{$\begin{array}{l}\text { Média } \\
42,16\end{array}$} & \multicolumn{2}{|c|}{ IC $95 \%$} & \multirow{3}{*}{$\begin{array}{l}\text { Média } \\
36,00\end{array}$} & \multicolumn{2}{|c|}{ IC $95 \%$} & & \\
\hline & & & Inf. & Sup. & & Inf. & Sup. & & Inf. & Sup. & & \\
\hline 1 & Funcionamento físico & & 30,72 & 49,38 & & 30,79 & 53,54 & & 19,69 & 52,31 & 0,3810 & 0,8488 \\
\hline 2 & Função física & 12,99 & 04,94 & 21,04 & 11,19 & 01,49 & 20,90 & 16,43 & 02,06 & 30,80 & 0,3886 & 0,8560 \\
\hline 3 & Dor & 55,59 & 46,24 & 64,94 & 58,28 & 46,68 & 69,89 & 50,43 & 34,71 & 66,15 & 0,2651 & 0,8572 \\
\hline 4 & Saúde geral & 52,60 & 46,81 & 58,38 & 51,49 & 44,84 & 58,15 & 54,71 & 43,60 & 65,83 & 0,4606 & 0,8575 \\
\hline 5 & Bem-estar emocional & 68,51 & 62,46 & 74,56 & 72,12 & 65,02 & 79,22 & 61,60 & 50,98 & 72,22 & 0,0197 & 0,8560 \\
\hline 6 & Função emocional & 20,59 & 10,32 & 30,86 & 17,91 & 06,08 & 29,74 & 25,71 & 06,12 & 45,31 & 0,3135 & 0,8518 \\
\hline 7 & Função social & 63,73 & 55,54 & 71,91 & 65,30 & 55,81 & 74,79 & 60,71 & 45,15 & 76,28 & 0,4583 & 0,8499 \\
\hline \multirow[t]{2}{*}{8} & Energia e fadiga & 61,18 & 55,30 & 67,05 & 61,79 & 54,90 & 68,69 & 60,00 & 48,98 & 71,02 & 0,6866 & 0,8502 \\
\hline & Escala ou Dimensão Específica & & & & & & & & & & & \\
\hline 9 & Lista de sintomas e problemas & 75,12 & 69,36 & 80,88 & 78,10 & 71,56 & 84,65 & 69,41 & 58,63 & 80,19 & 0,0435 & 0,8531 \\
\hline 10 & Efeitos da doença renal & 48,74 & 41,14 & 56,35 & 47,76 & 38,20 & 57,32 & 50,63 & 38,00 & 63,25 & 0,6181 & 0,8462 \\
\hline 11 & Sobrecarga da doença renal & 37,56 & 31,16 & 43,96 & 37,87 & 30,74 & 45,01 & 36,96 & 24,11 & 49,82 & 0,8510 & 0,8516 \\
\hline 12 & Papel profissional & 07,35 & 00,75 & 13,95 & 09,70 & 00,15 & 19,25 & 02,86 & 00,00 & 08,44 & $0,5825^{\star}$ & 0,8665 \\
\hline 13 & Função cognitiva & 78,56 & 71,76 & 85,36 & 82,79 & 75,04 & 90,54 & 70,48 & 58,05 & 82,90 & 0,0151 & 0,8551 \\
\hline 14 & Qualidade da interação social & 75,69 & 69,34 & 82,03 & 74,93 & 67,26 & 82,59 & 77,14 & 65,78 & 88,50 & 0,6436 & 0,8586 \\
\hline 15 & Função sexual & 75,42 & 66,83 & 84,00 & 76,39 & 63,75 & 89,03 & 73,96 & 64,34 & 83,57 & $0,2804^{*}$ & 0,8738 \\
\hline 16 & Sono & 64,79 & 58,04 & 71,54 & 65,19 & 56,64 & 73,73 & 64,02 & 52,95 & 75,10 & 0,8197 & 0,8599 \\
\hline 17 & Suporte social & 74,18 & 64,37 & 84,00 & 74,13 & 62,01 & 86,24 & 74,28 & 57,31 & 91,26 & 0,9832 & 0,8696 \\
\hline 18 & Estímulo por parte da equipe de diálise & 95,96 & 92,26 & 99,65 & 94,96 & 89,58 & 100,00 & 97,86 & 94,81 & 100,00 & $0,7351^{*}$ & 0,8710 \\
\hline 19 & Satisfação do paciente & 90,36 & 85,87 & 94,85 & 90,55 & 84,64 & 96,45 & 90,00 & 83,30 & 96,69 & 0,8711 & 0,8708 \\
\hline
\end{tabular}

Para o cálculo do p-valor foi utilizado o teste $t$ de Student, exceto nos valores com asterisco (*), neste caso foi utilizado o teste de

Mann-Whitney. 
DOI: $10.18605 / 2175-7275 /$ cereus.v13n2p176-192 Revista Cereus
SILVA, P.; LEÃO-CORDEIRO J.A.B.; ALMEIRA, R.J.; SILVA,

A.M.T.C.

Hemodiálise: análise da qualidade de vida de indivíduos renais crônicos.

Tabela 8. Dimensões do KDQOL-SF (Kidney Disease Quality of Life Short Form) estratificadas pelas variáveis socioeconômicas e clínicas dos 102 pacientes portadores de DRC na Clínica de Hemodiálise, Porangatu, Goiás, Brasil, 2019.

\begin{tabular}{|c|c|c|c|c|c|c|c|c|c|c|c|c|c|c|c|c|c|c|c|}
\hline \multirow{2}{*}{ Variáveis $(n=102)$} & \multicolumn{8}{|c|}{ Escala ou Dimensão Genérica } & \multicolumn{11}{|c|}{ Escala ou Dimensão Específica } \\
\hline & $1 \phi$ & 2 & 3 & 4 & 5 & 6 & 7 & 8 & 9 & 10 & 11 & 12 & 13 & 14 & 15 & 16 & 17 & 18 & 19 \\
\hline \multicolumn{20}{|l|}{ Idade (anos) } \\
\hline Até 59 anos & 54,62 & 17,79 & 64,95 & 50,00 & 69,62 & 26,28 & 66,83 & 64,04 & 77,32 & 53,43 & 38,22 & 9,62 & 77,44 & 74,74 & 83,70 & 67,42 & 75,64 & 96,63 & 89,42 \\
\hline$\geq 60$ anos & 24,90 & 8,00 & 45,85 & 55,30 & 67,36 & 14,67 & 60,50 & 58,20 & 72,83 & 43,88 & 36,88 & 5,00 & 79,73 & 76,67 & 48,21 & 62,05 & 72,67 & 95,25 & 91,33 \\
\hline$(p$-valor $)$ & $<0,0001$ & $0,1861^{*}$ & 0,0036 & 0,1999 & 0,6035 & 0,1128 & 0,2804 & 0,1641 & 0,2762 & 0,0389 & 0,7695 & $0,7328^{*}$ & 0,6379 & 0,6724 & 0,0055 & 0,2662 & 0,6728 & $0,7127^{*}$ & 0,5524 \\
\hline \multicolumn{20}{|l|}{ Renda Pessoal } \\
\hline$\leq 1 \mathrm{SM}$ & 38,37 & 12,35 & 53,98 & 51,63 & 66,89 & 20,08 & 60,99 & 60,42 & 73,57 & 47,44 & 35,92 & 4,22 & 77,03 & 73,25 & 75,48 & 64,53 & 72,09 & 96,23 & 88,96 \\
\hline$>1 \mathrm{SM}$ & 47,37 & 15,79 & 62,63 & 56,84 & 75,58 & 22,81 & 75,66 & 64,47 & 81,91 & 54,44 & 44,74 & 21,05 & 85,26 & 86,32 & 75,00 & 65,92 & 83,33 & 94,74 & 96,49 \\
\hline (p-valor) & 0,2941 & 0,6427 & 0,3142 & 0,3268 & 0,1171 & 0,7733 & 0,0497 & 0,4536 & $0,1745^{\star}$ & 0,3165 & 0,1327 & 0,3064 & 0,1871 & 0,0236 & 0,9775 & 0,8228 & 0,2120 & $0,5164^{*}$ & $0,1500^{*}$ \\
\hline \multicolumn{20}{|l|}{ Escolaridade } \\
\hline Até Fundamental & 33,65 & 12,82 & 50,26 & 53,40 & 68,62 & 20,51 & 62,98 & 60,96 & 75,66 & 48,16 & 38,78 & 3,85 & 80,09 & 79,15 & 75,63 & 60,83 & 75,43 & 96,63 & 91,67 \\
\hline Médio ou Mais & 60,83 & 13,54 & 72,92 & 50,00 & 68,17 & 20,83 & 66,15 & 61,88 & 73,37 & 50,65 & 33,59 & 18,75 & 73,61 & 64,44 & 75,00 & 77,64 & 70,14 & 93,75 & 86,11 \\
\hline$(p$-valor $)$ & 0,0004 & 0,9156 & 0,0034 & 0,4870 & 0,9302 & 0,9706 & 0,6476 & 0,8542 & 0,6391 & 0,6981 & 0,3369 & $0,2609^{*}$ & 0,2590 & $0,0251^{*}$ & 0,9594 & 0,0026 & 0,5237 & 0,3553 & 0,1415 \\
\hline \multicolumn{20}{|l|}{$\begin{array}{l}\text { Hipertensão } \\
\text { Arterial }\end{array}$} \\
\hline Sim & 36,71 & 12,86 & 53,04 & 47,86 & 64,51 & 15,71 & 58,93 & 57,14 & 71,77 & 42,77 & 35,09 & 7,14 & 74,29 & 72,19 & 66,88 & 63,12 & 75,95 & 96,07 & 92,86 \\
\hline Não & 47,34 & 13,28 & 61,17 & 62,97 & 77,25 & 31,25 & 74,22 & 70,00 & 82,45 & 61,82 & 42,97 & 7,81 & 87,92 & 83,33 & 92,50 & 68,44 & 70,31 & 95,70 & 84,90 \\
\hline (p-valor) & 0,1384 & 0,9457 & 0,2592 & 0,0005 & 0,0055 & 0,0482 & 0,0142 & 0,0038 & $0,0264^{*}$ & 0,0009 & 0,1091 & 0,8957 & 0,0083 & 0,0213 & $0,0453^{*}$ & 0,3069 & 0,4568 & $0,3731^{*}$ & 0,0201 \\
\hline \multicolumn{20}{|l|}{ Diabetes Melitus } \\
\hline Sim & 26,71 & 5,26 & 50,92 & 45,39 & 62,42 & 10,53 & 52,96 & 53,16 & 66,12 & 37,58 & 32,89 & 5,26 & 68,25 & 68,77 & 58,65 & 58,38 & 71,05 & 98,68 & 93,42 \\
\hline Não & 47,97 & 17,58 & 58,36 & 56,88 & 72,13 & 26,56 & 70,12 & 65,94 & 80,47 & 55,37 & 40,33 & 8,59 & 84,69 & 79,79 & 88,24 & 68,59 & 76,04 & 94,34 & 88,54 \\
\hline (p-valor) & 0,0016 & $0,0349^{*}$ & 0,2826 & $0,0091^{*}$ & $0,0308^{*}$ & $0,0303^{*}$ & 0,0039 & 0,0027 & $0,0024^{\star}$ & 0,0012 & 0,1151 & 0,4963 & $0,0052^{*}$ & $0,0351^{*}$ & 0,0068 & 0,0392 & 0,4929 & $0,4023^{*}$ & 0,1411 \\
\hline \multicolumn{20}{|l|}{ Obesidade } \\
\hline Sim & 41,43 & 14,29 & 60,71 & 62,14 & 80,57 & 38,10 & 67,86 & 70,71 & 79,47 & 65,63 & 36,61 & 21,43 & 85,71 & 90,48 & 100,00 & 60,00 & 78,57 & 100,00 & 97,61 \\
\hline Não & 39,95 & 12,89 & 55,21 & 51,89 & 67,62 & 19,30 & 63,42 & 60,47 & 74,80 & 47,50 & 37,63 & 6,32 & 78,04 & 74,60 & 73,66 & 65,14 & 73,86 & 95,66 & 89,82 \\
\hline (p-valor) & 0,9109 & 0,9031 & 0,6784 & 0,2101 & 0,1295 & 0,1953 & 0,7026 & 0,2176 & 0,5679 & 0,0453 & 0,9102 & $0,3752^{*}$ & 0,4257 & $0,0415^{\star}$ & 0,0001 & 0,5913 & 0,7352 & $0,5166^{*}$ & $0,3507^{*}$ \\
\hline
\end{tabular}

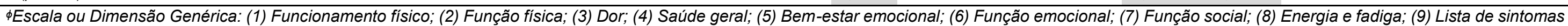

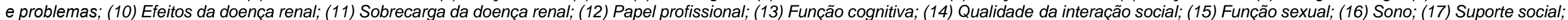
(18) Estímulo por parte da equipe de diálise; (19) Satisfação do paciente. Testes inferenciais: Teste de t de Student; $\left(^{*}\right)$ Teste de Mann-Whitney 


\section{DISCUSSĀO}

A taxa de prevalência global do tratamento dialítico tem crescido anualmente, sendo que, em 2016, 39.714 pacientes iniciaram tratamento. Desses, 48\% eram da região Sudeste; e para as demais regiões foram: Nordeste, com 19\%; Sul, com 17\%; CentroOeste, com 10\%; e Norte, com 5\%. A DRC requer tratamento de substituição renal, sendo que uma das modalidades é o processo hemodialítico, que gera dependência da máquina, como manutenção e prolongamento de vida (EVANGELISTA et al., 2018).

No presente estudo, evidenciou-se que houve prevalência de pacientes do sexo masculino, entre 19 e 59 anos, com renda pessoal de até 1 salário-mínimo e com, no máximo, o ensino fundamental concluído. De acordo Sesso et al. (2017), o sexo masculino (57\%) foi predominante, o que corrobora os achados de Miyahira et al. (2016), que tiveram $67,4 \%$ de homens no grupo avaliado.

A mortalidade, por insuficiência renal, é 10 a 20 vezes maior do que a da população em geral, mesmo por questões de idade, sexo, etnia e presença de diabetes mellitus, apesar da doença cardiovascular ser a causa mais comum de óbito (OLIVEIRA; ALVES; BEZERRA, 2009). Estudos epidemiológicos mostraram que a pressão arterial sistólica (PAS), a pressão arterial diastólica (PAD) e os fatores de risco tradicionais, para doenças cardiovasculares, estão associados a danos em órgãos-alvo, incluindo rigidez vascular e resultados ruins em pacientes em diálise (BUCHARLES et al., 2019). As principais comorbidades, percebidas neste estudo, foram: a hipertensão arterial e o diabetes mellitus.

Sesso et al. (2014) discutiram que o uso demasiado de medicações, em longo prazo, contribui para as desordens renais. Diante disso, na nossa pesquisa, evidenciamos que a grande maioria dos pacientes não utilizava os medicamentos que foram listados no momento da entrevista, como: atenolol, propranolol, captopril, enalapril, losartana, nifedipina, atensina, metformina, glibencamida, insulina, dentre outros. Porém, foram analisados os prontuários, posteriormente a entrevista, e foi constatado que os indivíduos entrevistados não sabiam quais medicamentos faziam uso contínuo. Após análise do prontuário, ficou evidente que a maioria dos pacientes utilizava: atensina, propranolol, atenolol, insulina NPH, regular e sustrate.

Apesar da porcentagem maioritária ter alegado não usar medicamentos, vale salientar que são pacientes que requerem cuidados sobre as prescrições de medicamentos, devido ao comprometimento da filtração e excreção dos rins (MARQUITO et al., 2014). 
Relacionado a doenças crônicas de base, geralmente, antecedentes familiares acabam acarretando outras doenças crônicas, como a insuficiência renal crônica (HALL; GUYTON, 2011). A maioria dos entrevistados apresentou casos de HAS na família, enquanto, sobre as outras patologias, como: diabetes mellitus, hipercolesterolemia, doença renal e obesidade, a maioria não admitiu casos familiares.

Pacientes com DRC estão sujeitos aos efeitos deletérios da inflamação, estresse oxidativo e toxinas urêmicas, refletindo em taxa de mortalidade cardiovascular de 20 a 30 vezes maior que a da população geral. Em adição a essa estatística, o hábito de fumar aumenta a incidência de insuficiência cardíaca, vasculopatia periférica e morte nesses pacientes (ELIHIMAS JÚNIOR et al., 2014).

O consumo de álcool é prejudicial à integridade do tecido renal, consequentemente, pode originar processos patológicos graves. O consumo de bebidas alcoólicas está diretamente associado ao desenvolvimento de insuficiência renal crônica, sendo um hábito importante a ser investigado nas anamneses clínicas (OLIVEIRA et al., 2011). Sobre os hábitos de tabagismo e etilismo, constatou-se, nesta pesquisa, que a maioria dos entrevistados afirmou não adotar tais práticas.

A prática de atividade física é considerada fator de proteção, capaz de atenuar as mudanças causadas pela doença e pelo tratamento, desacelerando a piora da função renal. Segundo alguns pesquisadores, a prática de atividade física aeróbia ou de resistência gera efeitos significativos na capacidade funcional, função muscular, performance física e qualidade de vida de pacientes com DRC (FUKUSHIMA; COSTA; ORLANDI, 2018). Sobre atividades físicas, apenas $23 \%$ dos entrevistados praticavam exercício físico regular.

O tratamento hemodialítico limita as atividades diárias, o que favorece o sedentarismo e a deficiência funcional, fatores que afetam diretamente a qualidade de vida. Em contrapartida, a realização de atividades físicas, por portadores de DRC, aumenta a capacidade funcional e tem efeito positivo sobre a qualidade de vida (MEDINA et al., 2010).

Entende-se que a hemodiálise, enquanto um procedimento de apoio à função renal, consiste na remoção de substâncias tóxicas e excesso de líquido por uma máquina de diálise, em procedimento com duração de 2 a 4 horas, exigindo que o paciente se desloque para a unidade de tratamento de 2 a 4 x/semana (BORGES; COSTA, 2019). Neste estudo, constatou-se que a maioria dos pacientes se deslocava de Van, 3 vezes por semana.

Tem sido demonstrado que, na medida em que ocorre a perda da função renal, há redução espontânea do consumo alimentar e, consequente, depleção do estado nutricional naqueles pacientes que nunca receberam orientação nutricional. Além disso, há evidências 
de que a pior condição nutricional no início do tratamento dialítico contribui para uma pior sobrevida ao longo dos anos. Neste contexto, esses pacientes não deixam dúvidas de que as abordagens nutricionais de recuperação ou manutenção de um bom estado nutricional são necessárias já nos estágios iniciais da DRC (CUPPARI; KAMIMURA, 2009).

Segundo Travagin et al. (2010), a progressão da DRC pode ser identificada por meio dos níveis de TFG. Diante disso, o profissional de saúde pode conduzir uma avaliação criteriosa com base nessa taxa para verificar a evolução da doença. Assim, é necessário oferecer orientações sobre a alimentação adequada e os exercícios físicos que ajudam tanto na prevenção como no retardo da progressão patológica. $\mathrm{Na}$ última versão das diretrizes sobre DRC, foi sugerido classificar a doença embasando-se na causa, no resultado da filtração glomerular e na albuminúria, o que possibilita reconhecer os riscos de desfechos adversos referentes ao comprometimento dos rins e ao óbito (KDIGO, 2012).

A maior frequência com que os pacientes consomem legumes e verduras foi superior a 3 vezes por semana. A frequência mais expressiva com que os pacientes consomem frutas foi a de até 3 vezes por semana. Diante do resultado, percebe-se que os pacientes contam com fonte satisfatória de nutrientes saudáveis, auxiliando, assim, na terapêutica.

Quando uma pessoa é acometida por uma doença de características crônicas, enfrenta diversas alterações no estilo de vida, provocadas por inúmeras restrições decorrentes da sua presença, das necessidades terapêuticas e de controle clínico, além da possibilidade de submeter-se a internações hospitalares recorrentes (OLIVEIRA; ALVES; BEZERRA, 2009).

Diante disso, o estudo identificou que dentre as mudanças estabelecidas no formulário, as mais impactantes foram percebidas no trabalho e nas atividades do dia a dia. Então, a doença renal pode exercer mudança brusca, principalmente, na vida profissional do paciente. Portanto, os limites impostos pelo tratamento de hemodiálise, por alguns instantes, levam os indivíduos a enxergar uma rotina de tratamento sob uma perspectiva negativa, uma vez que acarreta comprometimento na efetivação nas atividades diárias, impactando, principalmente nas atividades laborais e domésticas (MARTINS et al., 2005).

Nesse estudo, o emprego do instrumento KDQOL-SF possibilitou a avaliação da qualidade de vida de indivíduos com DRC em hemodiálise. Esse instrumento apresentou dados para análise tanto de dimensões genéricas como de específicas da doença renal.

Diante das dimensões específicas do KDQOL-SF, o maior escore médio obtido foi em: estímulo por parte da equipe de diálise $(95,96)$, correlacionando com os resultados dos 
autores Medeiros et al. (2015) e Franco et. al. (2011), em que obtiveram como maior escore médio a mesma dimensão.

Lira, Avelar e Bueno (2015) obtiveram, como maior escore: a função sexual, visto que nos resultados dos autores Medeiros et al. (2015) e Franco et al. (2011), esta dimensão estava em segundo lugar como maior escore. No presente estudo, esta dimensão ficou em quinto lugar, destacando que as principais foram estímulo por parte da equipe de diálise $(95,96)$, satisfação do paciente $(90,36)$, função cognitiva $(78,56)$, qualidade da interação social $(75,69)$, função sexual $(75,42)$ e lista de sintomas e problemas $(75,12)$.

Evangelista et al. (2018) destacaram que, em pacientes com estágio final da doença renal, há uma diminuição exacerbada na escala de componente físico do SF-36 em pacientes submetidos à hemodiálise, destacando as dimensões: função física e cognitiva como as mais afetadas. Além disso, afirmam que os portadores de DRC apresentam: sintomas físicos de fadiga e perda de energia; restrição da vida social; com os domínios funcionamento físico, dor e funcionamento social, no KDQOL-SF, com escores mais baixos.

No presente estudo, nas dimensões genéricas, os escores com melhor desempenho foram: bem-estar emocional $(68,51)$, função social $(63,73)$ e energia e fadiga $(61,18)$. Apesar de Evangelista et al. (2018) terem ressaltado que a função social, energia e fadiga e dor, geralmente, apresentam escores baixos, neste estudo não foi observado tal evento. Entretanto, como menores escores tivemos: funcionamento físico $(40,05)$, função emocional $(20,59)$ e função física $(12,99)$, estando de acordo com o que Evangelista et al. (2018) descreveram, ao destacar que a função física é uma das mais prejudicada.

\section{CONSIDERACOÓES FINAIS}

O perfil dos pacientes renais crônicos, que fizeram parte deste estudo, foi, em sua maioria: indivíduos do sexo masculino, que contava com até um salário-mínimo, com escolaridade restrita ao ensino fundamental. Adicionalmente, a maior parte apresentava hipertensão e diabetes e usava alguma medicação, especialmente Losartana. Além disso, a maioria não fumava, não bebia e, também, não praticava exercícios físicos regulares. Eram pacientes que tinham o SUS como custeador da sua terapia de substituição renal. Alegaram ter ciência sobre sua saúde e sobre a função da hemodiálise, e perceberam mudanças em seus estilos de vida, após o diagnóstico da doença, especialmente, no trabalho, nas atividades do dia a dia e em viagens. 
A qualidade de vida se mostrou bastante afetada principalmente nas dimensões "papel profissional" e "função física". Por outro lado, as dimensões que sofreram menor impacto foram "estímulo por parte da equipe de diálise" e "satisfação do paciente". Os fatores sociodemográficos e clínicos associados à qualidade de vida do paciente renal crônico, em hemodiálise, que mais apresentaram significância foram: diabetes mellitus e hipertensão arterial sistêmica.

É de grande relevância que a DRC seja diagnosticada precocemente para que sejam implementadas medidas de promoção e prevenção à saúde da comunidade. Os dados apresentados aqui podem auxiliar na prática de profissionais de saúde, colaborando para o melhor entendimento e domínio dos aspectos que aumentam ou diminuem a qualidade de vida dos indivíduos em hemodiálise.

\section{REFERÉNCIAS}

BETTONI, Loren Caroline; OTTAVIANI, Ana Carolina; ORLANDI, Fabiana Souza. Associação entre o autocuidado e a qualidade de vida de pacientes com doença renal crônica. Rev. Eletr. Enf., v. 19, n. 14, p. 1-9, 2017.

BORGES, Daniela da Silva; COSTA, Tainara. Pacientes com insuficiência renal crônica e o estado nutricional. Rev. Unilago, v. 1, n. 1, p. 1-10, 2019.

BUCHARLES, Sérgio Gardano et al. Hipertensão em pacientes em diálise: diagnóstico, mecanismos e tratamento. J. Bras. Nefrol., São Paulo, v. 41, n. 3, p. 400-411, 2019.

CUPPARI, Lilian; KAMIMURA, Maria Ayako. Avaliação nutricional na doença renal crônica: desafios na prática clínica. J. Bras. Nefrol., v. 31, supl. 1, p. 28-35, 2009.

DIPP, Thiago et al. Quality of life as a predictor of hospitalization in patients with chronic kidney disease on hemodialysis: a retrospective cohort study. Clin. Biomed. Res., v. 39, n. 3, p. 209-215, 2019.

DUARTE, O.S; CICONELLI, R.M; SESSO, R. Cultural adaptation and validation of the "Kidney Disease and Quality of Life - Short Form (KDQOL-SF $\left.{ }^{T M} 1.3\right)$ " in Brazil. Braz. J. Med. Biol. Res., v. 38, n. 2, p. 261-270, 2005.

DUARTE, P. S. et al. Tradução e adaptação cultural do instrumento de avaliação de qualidade de vida para pacientes renais crônicos (KDQOL-SF). Revista da Associação Médica Brasileira, v. 49, n. 4, p. 375-381, 2003.

ELIHIMAS JÚNIOR, Ubiracé Fernando et al. Tabagismo como fator de risco para a doença renal crônica: revisão sistemática. J. Bras. Nefrol., v. 36, n. 4, p. 519-528, 2014.

EVANGELISTA, Renata Alessandra et. al. Domínios afetados na qualidade de vida do paciente renal crônico em tratamento hemodialítico: revisão sistemática. Rev. Enferm. Atenção Saúde, v. 7, n. 3, p. 150-164, 2018. 
FRANCO, L. Cunha et. al. Avaliação da qualidade de vida de portadores de insuficiência renal crônica em diálise renal. Enfermería Global, v. 10, n. 23, p. 165-171, 2011.

FUKUSHIMA, Raiana Lídice Mór; COSTA, José Luiz Riani; ORLANDI, Fabiana de Souza. Atividade física e a qualidade de vida de pacientes com doença renal crônica em hemodiálise. Fisioter. Pesqui., v. 25, n. 3, p. 338-344, 2018.

GOUVEIA, Denise Sbrissia e Silva et. al. Análise do impacto econômico entre as modalidades de terapia renal substitutiva. J. Bras. Nefrol., v. 39, n. 2, p. 162-171, 2017.

GUEDES, K.D; GUEDES, H.M. Qualidade de vida do paciente portador de insuficiência renal crônica. Rev. Ciência \& Saúde, Porto Alegre, v. 5, n. 1, p. 48-53, 2012.

HALL JE; GUYTON E. Tratado de fisiologia médica. 12. ed. Rio de Janeiro: Elsevier, 2011.

HAYS, R.D. et al. Kidney disease quality of life short form (KDQOL-SF) Version 1.3: a manual for use and scoring. Santa Monica, CA: RAND, 1997.

KDIGO 2012 Clinical Practice Guideline for the Evaluation and Management of Chronic Kidney Disease. Kidney Int (Suppl) 2013;3:1-150.

MAFRA, Vanderson Ramos et al. Prevalence of Disease Resulting from Chronic Renal Failure in Patients Treated in Pró-Rim Foundation in the Municipality of Gurupi-To. International Journal of Advanced Engineering Research and Science (IJAERS), v. 6, n. 10, p. 2456-1908, 2019.

MARQUITO, A.B. et al. Identifying potential drug interactions in chronic kidney disease patients. Jornal Brasileiro de Nefrologia, v. 36, n. 1, p. 26-34, 2014.

MARTINS M.R.I. et al. Qualidade de vida de pessoas com doença renal crônica em tratamento hemodialítico. Rev Latinoam Enferm. 2005.

MEDEIROS, Raquel Campos de et. al. Qualidade de vida relacionada à saúde de indivíduos em hemodiálise. Rev. Enferm., Recife, v. 9, supl. 9, p.1018-27, 2015.

MEDINA, Luiz Antonio Rodrigues et al. Atividade física e qualidade de vida em pacientes com doença renal crônica submetidos à hemodiálise. ConScientiae Saúde, v. 9, n. 2, p. 212-219, 2010.

OLIVEIRA, Adriene Soares de et al. Efeitos do alcoolismo crônico na morfologia renal de ratos Wistar. Rev. Bras. Clin. Med., São Paulo, v. 9, n. 1, p. 46-49, 2011.

OLIVEIRA, Fernanda Celedonio de; ALVES, Maria Dalva Santos; BEZERRA, Aline Pontes. Co-morbidades e mortalidade de pacientes com doença renal: atendimento terceirizado de nefrologia. Acta Paul. Enferm., v. 22, p. 476-480, 2009.

SESSO, Ricardo Cintra et al. Brazilian Chronic Dialysis Survey 2016. Jornal Brasileiro de Nefrologia, v. 39, n. 3, p. 229-245, 2017.

SESSO, Ricardo Cintra et al. Inquérito brasileiro de diálise crônica 2013: análise das tendências entre 2011 e 2013. Jornal Brasileiro de Nefrologia, v. 36, n. 4, p. 476-81, 2014. 
A.M.T.C.

Hemodiálise: análise da qualidade de vida de indivíduos renais crônicos.

TRAVAGIN, Darlene Suellen Antero et al. Prevenção e progressão da doença renal crônica: atuação do enfermeiro com diabéticos e hipertensos. Rev. Enferm. UERJ, Rio de Janeiro, v. 18, n. 2, p. 291-297, 2010. 